https://doi.org/10.15407/ujpe64.7.554

K. CIEŚLA, R. STASZEWSKI, J.J. CHWASTOWSKI

The Henryk Niewodniczański Institute of Nuclear Physics, Polish Academy of Sciences

(152, Radzikowskiego, 31-342 Kraków, Poland; e-mail: krzysztof.ciesla@ifj.edu.pl,

rafal.staszeski@ifj.edu.pl, janusz.chwastowski@ifj.edu.pl)

\title{
ON THE CENTRALITY DETERMINATION WITH FORWARD PROTON DETECTORS
}

\begin{abstract}
The forward proton detectors, already installed at the Large Hadron Collider, are studied in the context of heavy-ion collisions. The potential of such detectors in measuring the nuclear debris coming from the spectator fragments is presented. The geometric acceptance of the forward proton detectors for different debris is estimated. The impact of experimental conditions and the Fermi motion on the acceptance is studied. A possibility of the collision impact parameter reconstruction from the measurement of nuclear fragments is discussed.
\end{abstract}

Ke ywords: heavy-ion physics, impact parameter, forward detectors.

\section{Introduction}

The Large Hardon Collider [1] is equipped with forward proton detectors designed to register protons scattered in diffractive or electromagnetic interactions. Such protons are scattered at very low angles, and this requires detectors to be installed very far away from the interaction point. In addition, with the use of the Roman Pot technology, they can be placed very close to the beam. The LHC physics programme is not entirely devoted to studies of proton-proton interactions. The machine may also accelerate the heavy-ion beams. This resulted in many measurements of proton-lead and lead-lead collisions [2].

A sketch of an typical heavy-ion collision is shown in Fig. 1. It is quite obvious that the impact parameter of a collision has usually a non-zero value. This means that only a part of nucleons belonging to the one nucleus interacts with a part of nucleons of the other one. Nucleons actively participating in the interactions are called participants, in contrary to the spectators.

The time scale of a ultrarelativistic heavy-ion collision is much shorter than the time scale of the interactions within the nuclei. The spectators are mostly left intact and are scattered into the beampipe escaping the central detector acceptance, similar to the forward protons. This paper tries to answer whether

(C) K. CIEŚLA, R. STASZEWSKI,

J.J. CHWASTOWSKI, 2019

554 and to what extent the forward proton detectors installed at the LHC can be used with heavy-ion beams.

\section{Forward Proton Detectors}

Several systems of forward proton detectors are currently installed at the LHC, including: AFP [3], ALFA [4], CT-PPS [5], and TOTEM [6]. All of them are installed about $200 \mathrm{~m}$ away from their corresponding interaction points. The ALFA detectors approach the beams vertically, the AFP and CT-PPS horizontally, while TOTEM has both types of the detectors. This work takes the AFP detectors as an example. However, similar results could also be expected for other horizontal detectors.

The AFP (ATLAS Forward Proton) detectors [3] are a subsystem of the ATLAS experiment. They consist of four detector stations - two on each outgoing beam, with the near stations placed at $205 \mathrm{~m}$ and the far stations at $217 \mathrm{~m}$ away from the interaction point. Each AFP station includes four planes of 3D

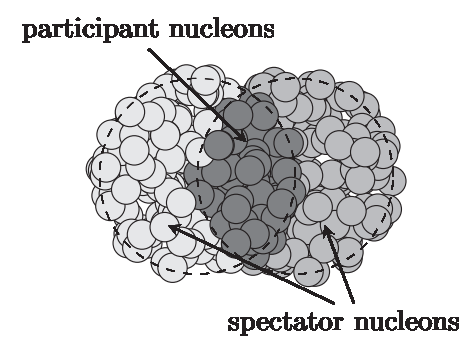

Fig. 1. Schematic diagram of an ultrarelativistic collision of two heavy nuclei (a view along the motion axis)

ISSN 2071-0194. Ukr. J. Phys. 2019. Vol. 64, No. 7 


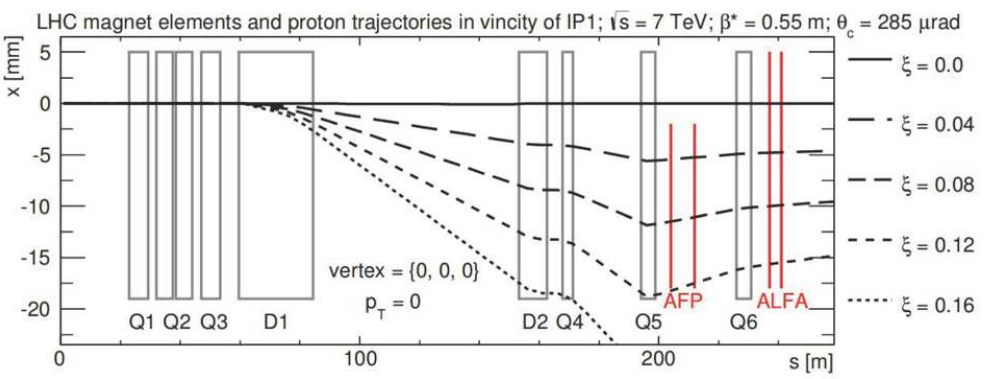

Fig. 2. Trajectories of forward protons in the LHC magnetic lattice. $s$ is the distance from the interaction point along the nominal optics, $x$ is the horizontal coordinate of a trajectory with respect to the nominal orbit, $\xi$ is the relative energy loss of a forward proton

Silicon pixel tracker sensors [7], the far stations are additionally equipped with quartz Cherenkov timeof-flight detector [8] (not important in the current study). The use of the Roman Pot mechanism allows the AFP detectors to be horizontally inserted into the accelerator beampipe.

Since the AFP detectors are located at some distance from the interaction point, the scattered protons, before being registered by them, travel through magnetic fields of several LHC magnets, confront Fig. 2. The Q1-Q3 quadrupole magnets are responsible for the final focusing of a beam, providing the high luminosity of the collisions. As for two dipole magnets, D1 separates the outgoing and the incoming beams, and D2 accommodates them within the beampipes. The Q4 and Q5 quadrupole magnets are used to match the interaction region optics to the optics of the rest of the machine.

As a result of the interaction, the forward protons produced in pp interactions (e.g., in diffractive processes) have slightly different kinematics, than the beam protons. They are scattered at a very small angle and often lose some part of their energy. Small scattering angles mean a very steep distribution of forward proton transverse momenta. Thus, the forward proton trajectory and the position in the detectors are primarily determined by the proton energy. The transverse momentum leads to a moderate smearing of the scattered proton position at the detector. The forward proton relative energy loss is defined as $\xi=1-E_{\text {proton }} / E_{\text {beam }}$. The higher its value, the larger is the forward proton trajectory curvature in the magnetic fields. An example of various trajectories is illustrated in Fig. 2, where also the positions of the LHC magnets and AFP detectors are

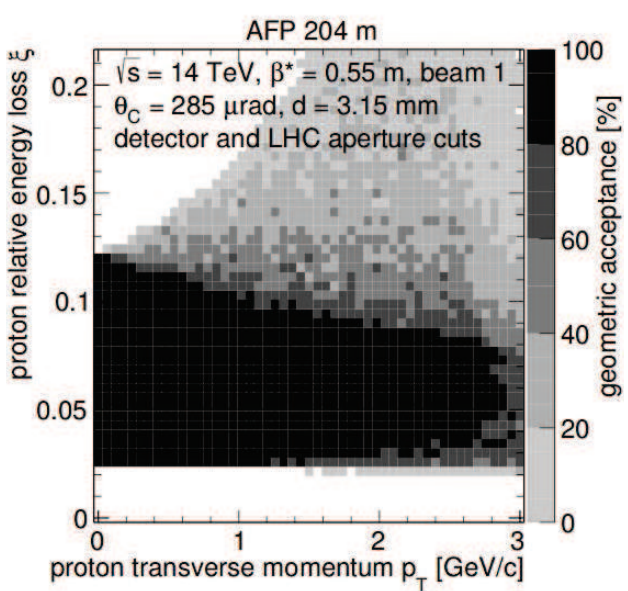

Fig. 3. Geometric acceptance of the AFP detectors for forward protons

shown. The kinematic range in which the measurements are possible can be described by the value of the geometric acceptance treated as a function of the relative energy loss and transverse momentum of the forward proton, $p_{T}$. The acceptance calculated using the MAD-X program [9] for the design LHC optics [10] is shown in Fig. 3. One can see that the AFP detectors can register protons with relative energy loss between $2 \%$ and $12 \%$ and less than $3 \mathrm{GeV}$ of the transverse momentum.

\section{Acceptance for Nuclear Spectator Fragments}

Before the ultrarelativistic heavy-ion collision, both participating and spectating nucleons are parts of the nucleus, interacting with each other. During an interaction, the spectators are, in a sense, peeled away from participants, and their ensemble is left in a very 


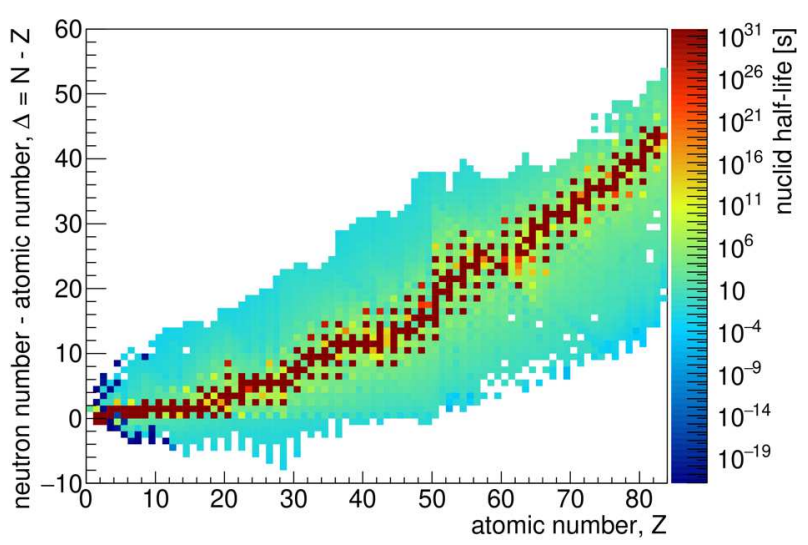

Fig. 4. Half-life time of the known nuclei

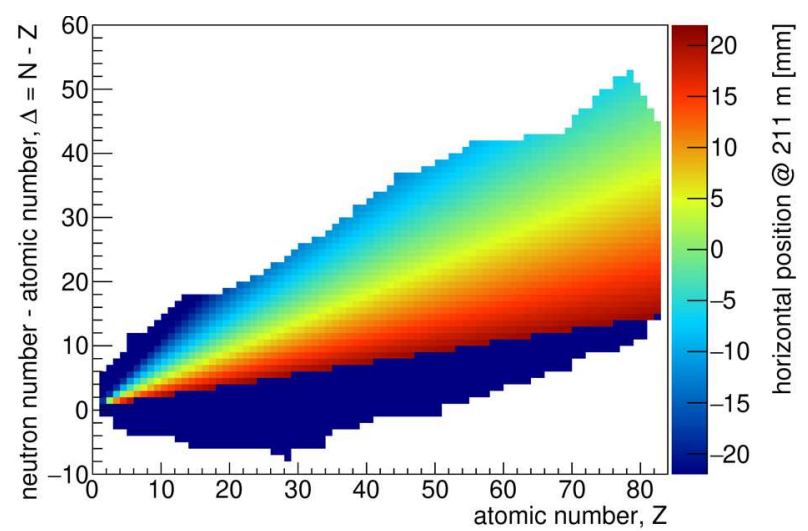

Fig. 5. Horizontal position of the nuclei at the distance of $211 \mathrm{~m}$ from the ATLAS interaction point

peculiar state which subsequently decays into lighter fragments. In the calculations of the geometric acceptance of the detectors for the spectator fragments, it is assumed that all nuclei lighter than the projectiles can possibly be produced.

Since the AFP detectors are positioned far away from the interaction point, it is necessary to check whether and which produced fragments can hit a detector before the decay. For a $\mathrm{Pb}$ beam at the LHC with magnets set as for $6.5 \mathrm{TeV}$ proton optics, the proper time between the production and reaching a detector is about $0.3 \mathrm{~ns}$. The half-life time of the known nuclei, as a function of the atomic number $Z$ and the difference between the number of neutrons and the atomic number $\Delta$ is shown in Fig. 4. We can see that a vast majority of known nuclei could reach the detectors before the decay.
The nuclear fragment transport simulation was preformed using the MAD-X program, assuming the beam of fully ionized ${ }^{208} \mathrm{~Pb}$ ions accelerated to the energy of $2.56 \mathrm{TeV}$ per nucleon. The LHC optics corresponding to the LHC Run 2 heavy-ion operations [10] was used. Trajectories of ions different from $\mathrm{Pb}$ were simulated by scaling their momenta to the momentum of a lead ion that would have same trajectory in the magnetic filed as the tracked one. This scaling procedure is possible due to the dependence of the trajectory observed in the magnetic field on the ratio of the particle momentum to its charge.

Without introducing the spreads originating from the beam emittance and internal motion of nucleons, a nucleus with a given $A$ and $Z$ will hit the AFP detectors at a well-defined position. Dipole magnets bend the beam trajectory in the horizontal direction, so the $x$-coordinate of a fragment trajectory plays the major role in this consideration. It should also be noted that, for the safety reasons, the detectors are positioned at some distance with respect to the beam. An additional dead material of the Roman Pot floor has also been taken into account in the calculations.

Predicted horizontal positions of all nuclei at $211 \mathrm{~m}$ from the interaction point (in the middle between the near and far stations) are shown in Fig. 5. The position of ${ }^{208} \mathrm{~Pb}(\Delta=44)$ and all nuclei with the same $\Delta / Z$ ratio is equal to zero. Nuclei containing less neutrons per proton are deflected outside the LHC ring, similarly as the forward protons, and can be registered in the AFP detectors. Nuclei with more neutrons per proton are deflected toward the LHC center and escape the detection. Nuclei with $\Delta / Z$ ratio very different from that of lead can be lost in the LHC and not reach the AFP detectors.

Neglecting the internal motion of the nucleons within a nucleus, the energy of each nucleon is the same and equal to the energy of the beam divided by the mass number of the beam particles: $E_{N}=$ $=E_{\text {beam }} / A_{\text {beam }}$. Assuming that the spectator fragments are left intact during the collision, the energy of a spectator with mass number $A$ will be equal to $A \cdot E_{N}$. The internal motion of nucleons is introduced by applying the Fermi-gas model of a nucleus. In the rest frame of a nucleus, the density of nucleon states is given by $d n \sim p^{2} d p$. In the simulation, the absolute value of the momentum of each nucleon was randomly drawn from a quadratic dis- 
tribution between zero and the Fermi momentum of $250 \mathrm{MeV}$. Then the momentum of a given fragment was calculated as a vector sum of the momenta of all its nucleons and the Lorentz-transformed into the laboratory frame.

For the beam emittance value of $1.233 \mu \mathrm{m}$ [10], the lead beam angular spread is $24 \mu \mathrm{rad}$ at the interaction point, and the interaction vertex distribution has the transverse and longitudinal spreads of $13 \mu \mathrm{m}$ and $5.5 \mathrm{~cm}$, respectively. The horizontal width of the beam at $211 \mathrm{~m}$ from the interaction point, $\sigma_{x}$, is $134 \mu \mathrm{m}$. This width is an usual unit of distance between the detector and the beam. The distance between the edge of a sensor and the center of the beam is assumed to be $3 \mathrm{~mm}$. It covers about $19 \sigma_{x}$ and a 0.5-mm-long distance between the active sensor edge and the outer wall of the Roman Pot floor.

The result of studies of the influence of various factors on the positions of the selected ions at the distance of $211 \mathrm{~m}$ away from the interaction point is shown in Fig. 6. One can observe that the effects of the beam spreads and those due to the transverse component of the Fermi motion are small. The position smearing is dominated by the longitudinal Fermi motion magnified by the Lorentz boost. This effect is stronger for the lighter nuclei.

Figure 7 shows the acceptance to detect a given nuclear fragment as a function of its $Z$ and $\Delta$ at a distance of $211 \mathrm{~m}$ away from the interaction point. The results were averaged over the distributions of the momenta and the Gaussian spatial and angular spreads of the LHC beam. However, the AFP detectors were not designed for measurement of the nuclear debris, and their acceptance covers a significant part of the nuclei spectrum. As one can observe, for a given $Z$, especially for heavier nuclei, more than a half of known nuclei can be potentially detected. This range of accepted masses decreases linearly with decreasing value of $Z$.

\section{Centrality Determination}

To study how the measurements of fragments can be used to retrieve information about a central state, a simulation of $\mathrm{Pb}-\mathrm{Pb}$ collisions using the DPMJET Monte Carlo generator [11] was performed. For each event, the generator reports a list of produced particles, including the spectators. Figure 8 shows the distribution of produced fragments.

ISSN 2071-0194. Ukr. J. Phys. 2019. Vol. 64, No. 7
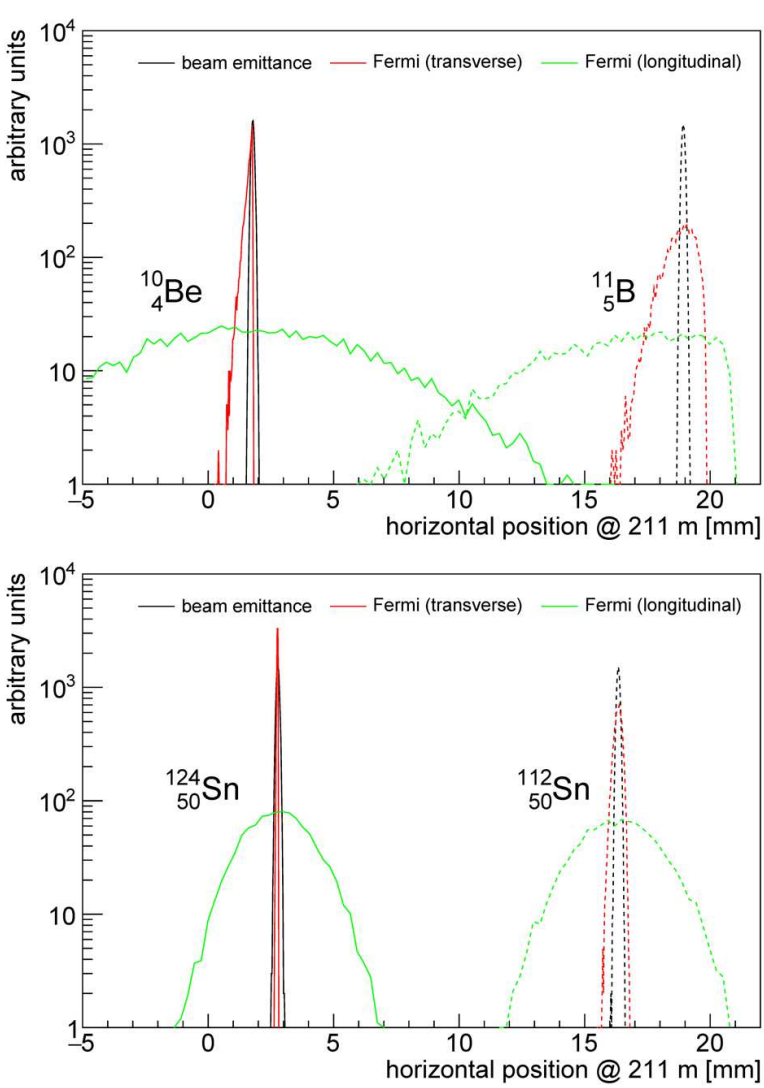

Fig. 6. Effects of the beam emittance and the Fermi motion on the fragment position in the AFP for selected light (top) and heavy (bottom) nuclei

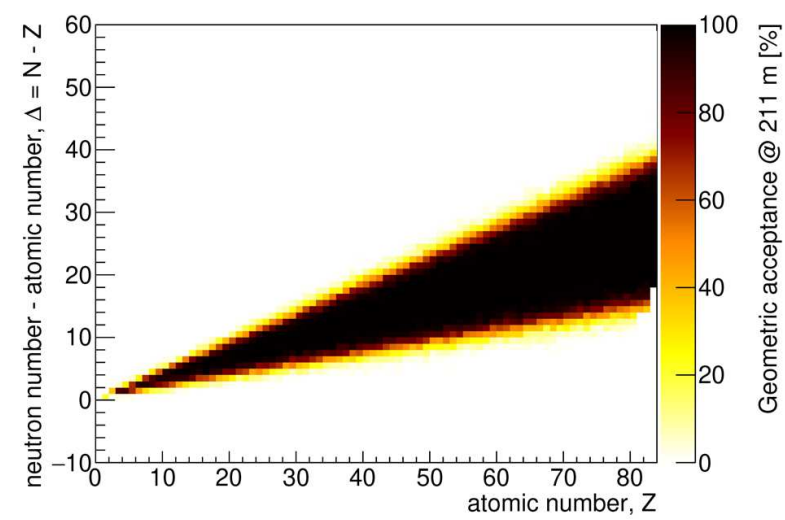

Fig. 7. AFP acceptance for nuclear fragments

Each event is generated with a known random value of the impact parameter. Figure 9, $a$ shows the correlation between the impact parameter value and the sum of the mass numbers of all produced frag- 


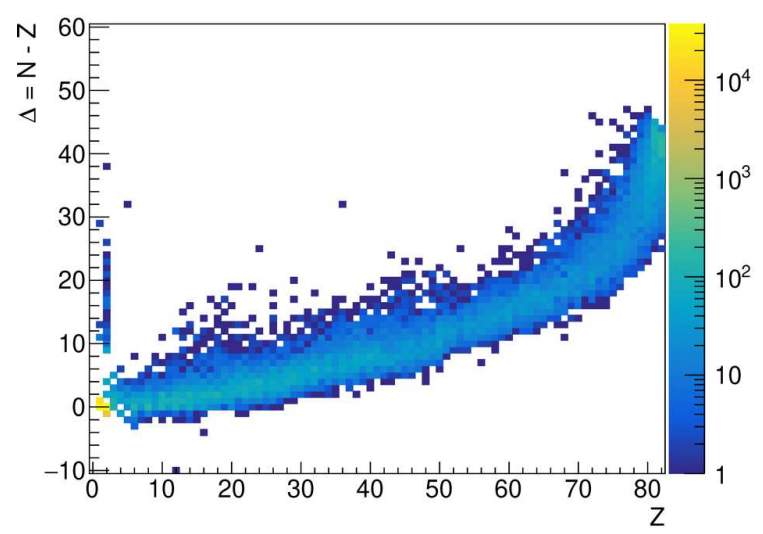

Fig. 8. Multiplicity of the nuclear fragments produced in $\mathrm{Pb}-$ $\mathrm{Pb}$ collisions simulated using DPMJET
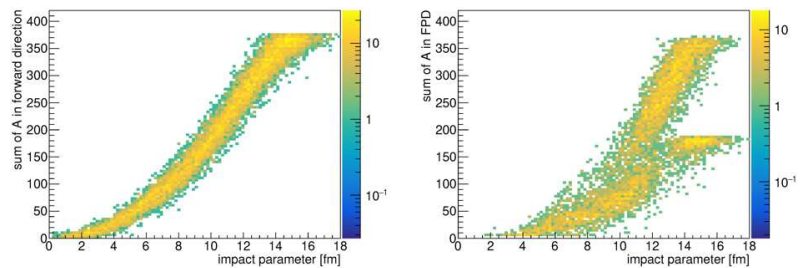

Fig. 9. Correlation between the collision impact parameter and the sum of mass numbers of: all produced fragments (left), fragments produced within the acceptance of forward proton detectors (right)

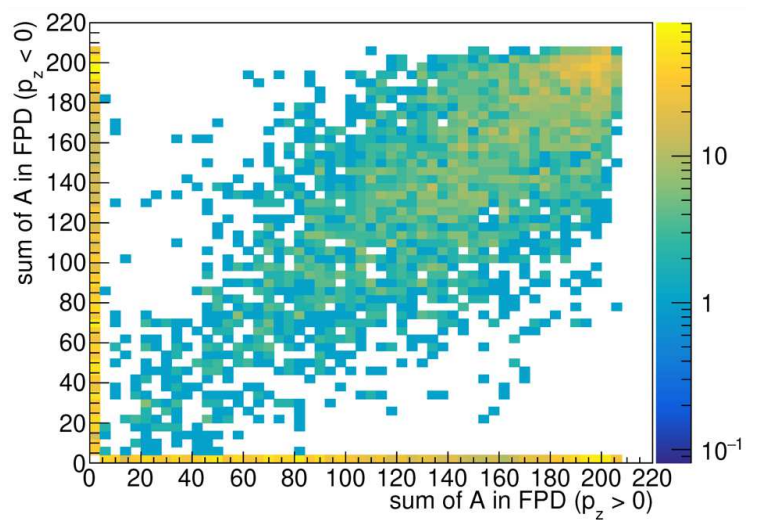

Fig. 10. Correlation between the sum of masses of fragments produced within the acceptance of forward proton detectors on the sides with positive and negative longitudinal momenta

ments. One can observe a strong dependence between these two variables - the more peripheral the event, the more the spectators are produced. Calculating this plot, the acceptance of forward proton detectors was not considered. Figure 9, $b$ shows the same correlation. But here, the sum runs only over the frag-

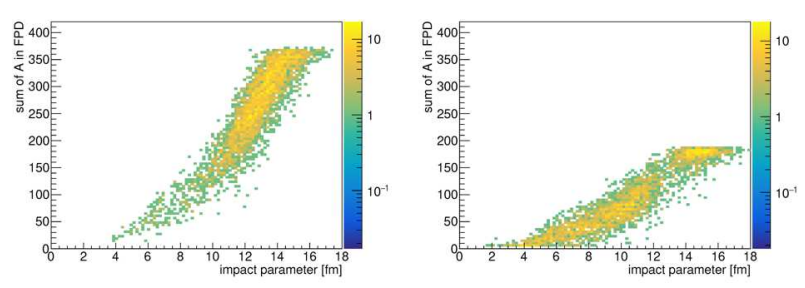

Fig. 11. Correlation between the collision impact parameter and the sum of mass numbers of fragments produced within the acceptance of forward proton detectors for double-tag (left) and single-tag (right) (see text)

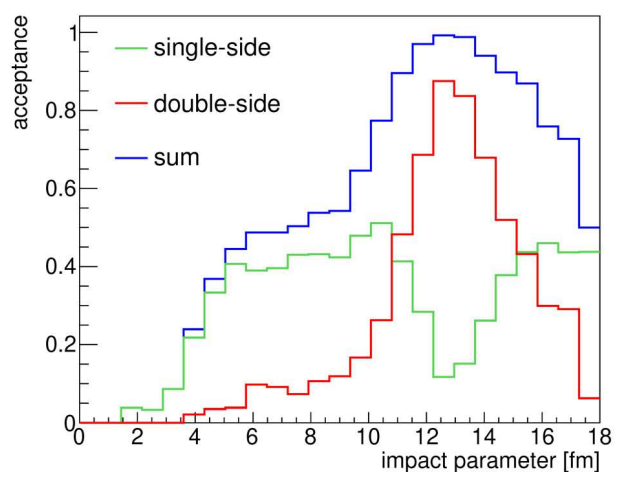

Fig. 12. Acceptance for events with nuclear fragments measured in forward proton detectors

ments within the acceptance of the forward proton detectors ${ }^{1}$. One can notice that the correlation persists. However, it is not as strong as in the previous case. The correlation has two components - one similar to the original one and the another one with $\Sigma A$ scaled down.

The initial state of the $\mathrm{Pb}-\mathrm{Pb}$ collision is, in the first approximation, symmetric with respect to the $p_{z}$ sign. In a particular event, fluctuations of the shape of ions and those related to the spectator fragmentation can break this symmetry leading to a twocomponent picture. Indeed, this can be observed from Fig. 10 showing the correlation between the sum of $A$ measured on the two sides. Two types of events can be distinguished - events with fragments on both sides (double-tag) and only one side (single-tag). For double-tag events, the $\Sigma A$ of the fragments measured on both sides are correlated. The width of this correlation reflects the correlation between the impact parameter and the measurements on each side.

${ }^{1}$ For the results based on the DPMJET simulation, the acceptance of the forward proton detectors is taken into account in an approximate way based on their $A / Z$ ratio. 
Figure 11 shows the correlations between the collision impact parameter and the sum of $A$ for measured fragments of the two types of events separately. A correlation between the two variables is visible in both cases, which shows that the proposed method can be used for the centrality determination.

Figure 12 shows the probability that a given event will be of either type as a function of the impact parameter. For the most central events (small impact parameter), the probability of observing any fragment in the forward detectors is zero. In such collisions, only the lightest fragments can be produced, which will escape the detection. With increasing value of the impact parameter, the probability of observing the single-tag events increases. However, at about 12$14 \mathrm{fm}$ it drops down, which corresponds to a peak in the probability for double-tag events.

\section{Conclusions}

The presented study shows that the existing forward proton detectors at the LHC provide an interesting possibility of detecting the nuclear debris originating from the collision of two heavy ions. One of the possibility is a measurement of the centrality of $\mathrm{Pb}-\mathrm{Pb}$ collisions. Different centralities result in different signals generated by the produced nuclear fragments. Such measurement would be independent of and complementary to the other commonly used methods. A direct measurement of the number of spectator fragments and, hence, the determination of the number of participants could be possible, if several forward detectors providing thus a much larger acceptance are used [12]. The present work shows that one can get information about the centrality even with the limited acceptance of the already existing detectors. More details can be found in [13].

The measurements of spectators using the Roman Pot detectors could be considered also for proton-ion and lepton-ion interactions. However, this topic requires dedicated studies.

This work was supported in part by a Polish National Science Center grant No. 2015/19/B/ST2/ 00989 .

1. L. Evans, P. Bryant. LHC machine. JINST 3, S08001 (2008).

2. F. Antinori, A. Dainese, P. Giubellino, V. Greco, M.P. Lombardo, E. Scomparin. Proc., 27th International
Conference on Ultrarelativistic Nucleus-Nucleus Collisions (Quark Matter 2018): Venice, Italy, May 14-19, 2018. Nucl. Phys. A 982, 1 (2019).

3. ATLAS Collaboration. Technical Design Report for the ATLAS Forward Proton Detector. CERN-LHCC-2015009, ATLAS-TDR-024.

4. ATLAS Collaboration. ATLAS Forward Detectors for Measurement of Elastic Scattering and Luminosity. CERN-LHCC-2008-004, ATLAS-TDR-018.

5. CMS-TOTEM Collaboration. CMS-TOTEM Precision Proton Spectrometer. CERN-LHCC-2014-021, TOTEMTDR-003, CMS-TDR-13.

6. TOTEM Collaboration. The TOTEM experiment at the CERN Large Hadron Collider. JINST 3, S08007 (2008).

7. S. Grinstein, M. Baselga, M. Boscardin, M. Christophersen, C. Da Via et al. Beam test studies of 3D pixel sensors irradiated non-uniformly for the ATLAS forward physics detector. Nucl. Instrum. Meth. A 730, 28 (2013).

8. J. Lange et al.. Beam tests of an integrated prototype of the ATLAS forward proton detector. JINST 11, P09005 (2016).

9. $M A D$ - Methodical Accelerator Design. https:// $\operatorname{mad}$.web.cern.ch $/ \mathrm{mad} /$.

10. LHC Optics Web Home. http://cern.ch/lhcoptics.

11. S. Roesler, R. Engel, J. Ranft. The Monte Carlo event generator DPMJET-III. In: Advanced Monte Carlo for Radiation Physics, Particle Transport Simulation and Applications. Edit. by A. Kling, F.J.C. Baräo, M. Nakagawa, L. Távora, P. Vaz (Springer, 2001).

12. S. Tarafdar, Z. Citron, A. Milov. A centrality detector concept. Nucl. Instrum. Meth. A 768, 170 (2014).

13. R. Staszewski, K. Cieśla, J. J. Chwastowski. Forward proton detectors in heavy ion physics. In: $X X V$ Cracow EPIPHANY Conference on Advances in Heavy Ion Physics, 8-11 January (2019).

Received 08.07.19

K. Чешла, Р. Сташевскі, Й.Й. Хвастовскі

ПРО ВИЗНАЧЕННЯ ЦЕНТРАЛЬНОСТІ

ЗА ДОПОМОГОЮ ДЕТЕКТОРІВ ПРОТОНІВ, ЩО ВИЛІТАЮТЬ УПЕРЕД

$\mathrm{P}$ е $з$ ю м е

Вивчаються детектори протонів, що вилітають уперед, які вже встановлені на великому Адронному Колайдері, для дослідження зіткнень важких іонів. Демонструються можливості таких детекторів для вивчення продуктів поділу ядер - фрагментів спектаторів. Дана оцінка геометричного аксептансу детекторів протонів, що вилітають уперед, у випадку різних продуктів поділу. Вивчається вплив на аксептанс умов експерименту та руху Фермі. Обговорюється можливість реконструкції параметра зіткнень за допомогою вимірювання ядерних фрагментів. 